\section{$\underset{\substack{\text { hommes } \\ \text { \& migrations }}}{ }$}

\section{Hommes \& migrations}

Revue française de référence sur les dynamiques

migratoires

1299 | 2012

Musulmanes et féministes en Grande-Bretagne

\title{
La rentrée littéraire et le prix de la Porte Dorée
}

$4^{\mathrm{e}}$ édition

\section{Élisabeth Lesne}

\section{(2) OpenEdition \\ 1 Journals}

\section{Édition électronique}

URL : http://journals.openedition.org/hommesmigrations/1427

DOI : 10.4000/hommesmigrations. 1427

ISSN : 2262-3353

\section{Éditeur}

Musée national de l'histoire de l'immigration

\section{Édition imprimée}

Date de publication : 1 septembre 2012

Pagination : 128-132

ISSN : 1142-852X

\section{Référence électronique}

Élisabeth Lesne, "La rentrée littéraire et le prix de la Porte Dorée », Hommes \& migrations [En ligne],

1299 | 2012, mis en ligne le 01 septembre 2014, consulté le 22 septembre 2020. URL : http://

journals.openedition.org/hommesmigrations/1427 ; DOI : https://doi.org/10.4000/

hommesmigrations. 1427

Ce document a été généré automatiquement le 22 septembre 2020.

Tous droits réservés 


\title{
La rentrée littéraire et le prix de la Porte Dorée
}

\author{
$4^{\mathrm{e}}$ édition
}

Élisabeth Lesne

1 Après Alice Zeniter et Michaël Ferrier, Henri Lopes a reçu, le 6 juin dernier, le prix littéraire de la Porte Dorée pour son 8 roman, Une enfant de Poto-Poto. L'immigration continue d'inspirer les écrivains. Très belle rentrée sur ce thème avec des romans qui font voyager, de la réunification du Vietnam à l'Afrique post-coloniale en passant par la guerre d'Algérie, des conflits du Moyen-Orient aux révolutions arabes et à l'Europe en crise. Panorama.

\section{L'usage du monde}

2 Les Désorientés d'Amin Maalouf et Rue des voleurs de Mathias Énard ont pour toile de fond, voire pour matière, les tumultes du monde arabe et leurs répercussions sur l'Occident. Des romans qui emportent le lecteur. Avec Amin Maalouf, nous sommes au Liban, face à "la gigantesque, la retentissante débâcle historique de la civilisation qui est la nôtre". Il a fallu que Mourad, un ami de jeunesse, l'appelle à son chevet pour qu'Adam, le personnage principal des Désorientés, se décide à revenir en 2001 dans son pays natal. Même s'il n'est jamais nommé, on reconnaît le Liban qu'Amin Maalouf a quitté lui aussi en 1976, en plein conflit. Après quelque vingt-cinq ans d'absence, Adam ne retrouve pas le pays qu'il a connu et encore moins celui dont il a rêvé avec ses copains, le "cercle des Byzantins", des jeunes gens nombreux à avoir choisi l'exil. S'il arrive trop tard pour se réconcilier avec Mourad, l'“ami désadopté", lui vient l'idée de réunir tous les membres de la bande : Naïm, le juif émigré au Brésil ; Ramez, l'Arabe musulman entrepreneur prospère installé en Jordanie ; Ramsy, son ex-associé qui s'est retiré dans un monastère ; Nidal, devenu islamiste... Autant de destins qui permettent à Amin Maalouf d'écrire un roman aux analyses lumineuses, entre autres sur les "deux tragédies rivales", celle des juifs et celle des Palestiniens, et sur ce qui pourrait être les "deux calamités majeures" du xxle siècle: "l'islamisme radical et l'anti-islamisme 
radical". Mathias Énard, dans Rue des voleurs, ne craint pas non plus d'aborder les sujets chauds, une décennie plus tard, et un peu plus à l'ouest. Nous sommes en 2011, l'année des révolutions arabes et de la crise économique en Europe. Ici, pas de nostalgie, c'est l'énergie de la jeunesse qui irrigue en direct ce roman plein de fièvre et d'aventures. Lakhdar, Marocain de Tanger, a 17 ans quand il est mis à la porte de chez lui pour avoir approché de trop près sa cousine. Commence alors pour lui une vie d'errance jusqu'à ce qu'il soit embauché comme libraire par le Groupe pour la diffusion de la pensée islamique. Il ne devient pas islamiste pour autant, il aime trop lire des polars et la littérature arabe, boire des bières et regarder les filles - il a la culture d'Énard. Quelques petits boulots plus tard, Lakhdar se retrouve à Barcelone au moment des manifestations des indignés et constate: "l'Espagne était un pays africain comme les autres". À la fin du roman, ce jeune Tangerois dira encore - il a alors 20 ans : "Je suis ce que j'ai lu, ce que j'ai vu, j'ai en moi autant d'arabe que d'espagnol et de français." Une identité qui ne se limite pas au hasard de la naissance, c'est également ce qu'a toujours revendiqué Van, Vietnamien arrivé en France à 16 ans, en 1978, une des voix de Lame de fond, le dernier roman de Linda Lê (voir la critique de Mustapha Harzoune p. 152). De là où il est, dans un cercueil, Van soliloque pour tenter de définir son rôle dans l'accident qui a causé sa mort. Tour à tour lui font écho sa fille Laure, sa femme Lou, et Ulma, sa demi-sœur. Correcteur dans l'édition, "une face tournée vers l'Orient, l'autre vers l'Occident", Van se sentait citoyen de l'univers. "Pour lui, se souvient sa fille, c'était une chance d'avoir deux pôles." Un équilibre qu'est venu chambouler son amour fou pour Ulma, elle aussi partagée entre l'Asie et l'Europe.

\section{Histoires vraies}

Cinq écrivains ont puisé la matière de leur livre dans leur vie ou dans celle de leur famille sans passer par la fiction. C'est à son père disparu que Yassaman Montazami consacre son premier roman, Le Meilleur des jours. Behrouz ne faisait pas partie des Iraniens qui avaient dû fuir leur pays après la Révolution islamique de 1979. Il était venu à Paris dès la fin des années soixante étudier à la Sorbonne et cultiver sa passion pour Karl Marx. Drôle - "la vie avec lui semblait un éternel $1^{\mathrm{er}}$ avril" -, généreux et hospitalier, cet "éternel thésard" entraîne ses proches dans un tourbillon de fantaisie. Mais une question aux résonances actuelles hante ces pages : que reste-t-il des espoirs que portaient les Iraniens de sa génération en aspirant à l'avènement d'une démocratie laïque en Iran? L'Iran est aussi au cœur d'Elle joue de Nahal Tajadod. Venue en France à l'âge de 17 ans, Nahal Tajadod a vécu son enfance et son adolescence sous le régime du Shah. Elle s'en souvient en retraçant le parcours mouvementé de Sheyda, une actrice iranienne née en 1983, donc après la révolution. "Nous sommes toutes les deux des femmes (...), nous sommes là, nous respirons les mêmes odeurs, nous entendons les mêmes sons. Mais quelque chose nous sépare à jamais. Un peu plus de vingt ans : tout un monde." Pourtant, Sheyda non plus ne vit plus en Iran à la suite de ses démêlés avec la censure islamique. Tragiques sont les bribes de l'histoire familiale que colombe Schneck rassemble dans La Réparation, en allant aux États-Unis et en Israël avant de se décider à se rendre en Lituanie, pays de sa grand-mère maternelle, où $95 \%$ des juifs ont été tués entre 1941 et 1943 . Colombe Schneck veut tout savoir : le ghetto de Kovno en Lituanie, les camps en Estonie et en Allemagne, la mort de Salomé - la cousine de sa mère, dont elle a donné le prénom à sa fille -, la survie de ses tantes Raya et Macha, tout ce qu'ont tu les survivants... Elle en est persuadée, “quand je saurai, je n'aurai plus 
peur". La découverte de la vérité s'accompagne d'une belle maxime : “Toujours choisir la vie, toujours quand cela est possible repérer le plaisir."La Vie sans fards de Maryse Condé offre aussi une superbe leçon d'énergie. La Guadeloupéenne évacue en quelques lignes "l'approche à pas sournois de la vieillesse puis de la maladie". Son désir est de “cerner la place considérable qu'a occupée l'Afrique dans (son) existence et dans (son) imaginaire", et de revivre ses jeunes années sur le continent africain. De Paris, elle part en 1959 enseigner en Côte d'Ivoire. Elle ira ensuite en Guinée, au Ghana, en Angleterre et au Sénégal, découvrant la littérature africaine francophone et anglophone, ainsi que tout ce qui sépare les Antillais, les Africains et les Noirs américains : "Plus j'allais, plus je constatais que la Négritude n'était qu'un beau rêve. La couleur ne signifie rien." $\mathrm{Ne}$ s'intéressant guère à la politique, elle évoque toutefois les régimes chaotiques et les dictateurs postindépendance, tel Sékou Touré qui, tous les jours, "beau comme un astre dans ses grands boubous blancs, passait sur le front de mer, conduisant lui-même sa Mercedes 280 SL décapotée". Le texte s'arrête quand, mère de quatre enfants qu'elle élève seule - dans les années soixante! -, elle devient écrivain: "L'Afrique enfin domptée (...) ne serait plus que la matière de nombreuses fictions." Quelle force, quel parcours !Vassilis Alexakis, dans L'Enfant grec, revient sur ses années de formation par ses lectures. Après avoir subi une opération, il se déplace entre rêve et réalité, du jardin du Luxembourg tout proche au jardin perdu de son enfance dans le quartier Callithéa à Athènes, en ressuscitant les héros des livres qui ont enchanté ses jeunes années : Long John Silver, Don Quichotte, D'Artagnan, Cyrano, Robinson, Tarzan, Jean Valjean, Robin des Bois... Lui parviennent toutefois les cris des manifestants réunis place de la Constitution à Athènes. Son pays natal, avec la crise, a atteint une nouvelle dimension : "il est devenu une grande puissance maléfique", dont les jeunes rêvent de partir.

\section{En guerre}

4 C'est un destin extraordinaire que raconte Tierno Monénembo dans Le Terroriste noir. Celui d'un tirailleur sénégalais, par ailleurs Guinéen, échappé de la garnison allemande de Neufchâteau après s'être battu dans la Meuse, et retrouvé à moitié mort un jour de 1940 à la lisière de la forêt des Vosges. "Vous a-t-on dit qu'avant son arrivée à Romaincourt, personne n'avait jamais vu de nègre ?" demande Germaine Tergoresse au neveu du tirailleur venu, quelque soixante ans plus tard, assister à la cérémonie rendant hommage à son oncle, Addi Bâ. "Imposant malgré sa petite taille, souriant, bien mis, distingué", ce tirailleur séduit les dames, devient le chef du maquis local et organise les jeunes qui veulent fuir le STO. Mais l'ennemi le rattrape en décembre 43. Étonnant, la manière dont Monénembo décrit les hauts faits d'Addi Bâ et les haines séculaires de deux familles dans une langue truffée de régionalismes. "Même si nous traversons la Méditerranée, l'Algérie nous poursuivra", dit Octavio dans Le Couvre-feu d'octobre de Lancelot Hamelin (voir la critique de Mustapha Harzoune, p. 151). Cette phrase pourrait servir d'exergue à ce roman et à celui de Fabienne Jacob, L'Averse. Deux fictions qui évoquent la guerre d'Algérie en mêlant vie intime et grande histoire dans une narration subtile et sinueuse. Dans L'Averse, Tahar vit ses dernières heures, et l'Algérie dont il est parti à 15 ans en 1962, "pays jamais plus foulé, seulement refoulé", lui revient dans un flot d'images: les aubes dans le djebel et le camp militaire où il était la mascotte des soldats français, ses parents assassinés et Souad qu'il a rejetée à la porte de la caserne du Nord-Constantinois... L'écriture est sensuelle pour évoquer l'arrivée à Paris et la toute-puissance de la jeunesse qui "propulse son corps par les rues et les 
boulevards", nerveuse pour dire la rage de celui qui a si souvent adopté "le dos rond plutôt que la rébellion"... Le Couvre-feu d'octobre est une lettre fleuve d'Octavio. Une sorte de testament adressé à Judith, son amour d'enfance qu'il a laissé à Oran en 1955, pour aller étudier à Paris. Fin 1956, son frère aîné le rejoint en métropole, marié à Judith... Tout oppose ces deux frères : l'aîné devient flic à la Goutte d'Or et rejoint l'OAS, l'autre s'engage pour l'indépendance algérienne et découvre les ambiguïtés du Parti communiste puis les luttes fratricides entre le FLN et le MNA. Un roman à la fois lyrique et très documenté.

\section{Jeunes françaises d'aujourd'hui}

Des impatientes de Sylvain Pattieu commence dans un lycée de banlieue où se côtoient "des centaines d'enfants des pavillons et des barres, enfants d'immigrés de la première, de la deuxième ou de la troisième génération". Parmi les élèves, deux jeunes filles bien différentes : la grande gueule Bintou Masinka, qui préfère faire la fête avec ses copines qu'aller en cours, et la sérieuse Alima Sissoko, qui rêve d'intégrer Sciences Po, de voir "la génération black-blanc-beur à l'école". Renvoyées toutes les deux à la suite d'un incident idiot, elles se retrouvent caissières dans un grand magasin. Solidaires et toujours aussi impatientes d'en découdre. L'exil n'a rien d'une malédiction dans la plupart de ces romans, c'est plutôt le plus sûr moyen d'échapper aux "identités meurtrières", comme dirait Amin Maalouf, qui écrit aussi dans Les Désorientés: "Quitter son pays est dans l'ordre des choses; quelquefois, les événements l'imposent; sinon, il faut s'inventer un prétexte." Comme nous arrêtons notre sélection en mars, d'autres romans viendront biensûr compléter ce panorama. Nous restons à l'affût après cette première salve de qualité. Nous aurons l'occasion de revenir plus longuement sur certains titres et le plaisir d'inviter leurs auteurs aux cafés littéraires de la Cité.

\section{BIBLIOGRAPHIE}

Vassilis Alexakis, L'Enfant grec, Stock

Maryse Condé, La Vie sans fards, Stock

Mathias Énard, Rue des Voleurs, Actes Sud

Lancelot Hamelin, Le Couvre-Feu d'octobre, L'Arpenteur

Fabienne Jacob, L'Averse, Gallimard

Linda Lê, Lame de fond, Christian Bourgois

Amin Maalouf, Les Désorientés, Grasset

Tierno Monénembo, Le Terroriste noir, Seuil

Yassaman Montazami, Le Meilleur des jours, Sabine Wespieser

Sylvain Pattieu, Des impatientes, Le Rouergue 
Colombe Schneck, La Réparation, Grasset

Nahal Tajadod, Elle joue, Albin Michel

\section{AUTEUR}

\section{ÉLISABETH LESNE}

Responsable du prix littéraire de la Porte Dorée. 